\title{
Does Cultural Context Influence Descriptions of Change After a Near-Death Experience? Exploratory Findings From an Aotearoa New Zealand Sample
}

\author{
Natasha A. Tassell-Matamua, PhD; Kate L. Steadman, MA; \\ and Karen E. Frewin, PhD \\ Massey University
}

\begin{abstract}
A host of literature accumulated over the past 40 years attests to the powerfully transformative nature of near-death experiences (NDEs) through a consistently reported pattern of aftereffects. Many of the qualitative and quantitative studies of post-NDE changes have been conducted in the United States and in Western European nations. Although examining the cultural diversity of NDEs was recently highlighted as a research priority, no researchers to date have examined how near-death experiencers (NDErs) from Aotearoa New Zealand describe any changes they experience after their NDEs, whether these descriptions are similar to those in previous research with samples from other Western nations, and, if not, whether culturally-specific explanations might account for those cross-cultural differences. In this exploratory qualitative study, our aim was to examine these questions. Participants were 28 individuals who self-identified as NDErs. Four participants were interviewed about their NDEs, whereas a further 24 provided written accounts. All 28 accounts were thematically analyzed, with findings overall indicating that many of the NDE aftereffects reported in previous literature were also evident in our sample. In cases in which participants did not describe typical aftereffects identified in previous literature, possible culturally specific explanations are provided.
\end{abstract}

KEY WORDS: near-death experience, aftereffects, Aotearoa New Zealand, culture, cultural context

Natasha A. Tassell-Matamua, PhD, is a senior lecturer and Deputy Head of the School of Psychology, and Kate L. Steadman, MA, is a clinical psychology trainee, both with the School of Psychology, Massey University, Palmerston North, Aotearoa New Zealand. Karen E. Frewin, PhD, is a senior lecturer with the Institute of Education, Massey University, Palmerston North, Aotearoa New Zealand, and a registered counseling psychologist. Correspondence regarding this article should be sent to Dr. Tassell-Matamua at email: N.A.Tassell-Matamua@massey.ac.nz. 
Near-death experiences (NDEs) have often been described as powerfully transformative. Raymond Moody's book Life After Life (1975) provided the first literary information about NDE aftereffects, of which he noted the most common was loss of fear of death. The majority of other transformations were considered life changing and almost exclusively positive. Russell Noyes (1980) conducted the first systematic study of aftereffects, noting that of the 205 participants who had been in a life-threatening situation, nearly two-thirds reported life changes including loss of fear of death, strengthened belief in an afterlife, and a sense of importance or purpose. Empirical studies accumulated over the past 40 years have verified Moody's initial observations and Noyes's findings, in addition to providing consistent evidence of a pattern of frequently occurring aftereffects, irrespective of participant demographics or whether the NDE might be considered pleasurable-dominated by feelings such as love and peace-or distressing - dominated by feelings such as terror or anguish (Noyes, Fenwick, Holden, \& Christian, 2009; for overviews of distressing NDEs, see Atwater, 2009; Bush, 2002).

\section{NDE Aftereffects}

In their comprehensive analysis of research conducted since 1975, Noyes and colleagues (2009) categorized some of the commonly reported aftereffects of pleasurable Western NDEs into attitudinal and belief changes. They suggested three specific ways attitudes and beliefs change, which are summarized below.

\section{Changes in Perceptions of Self}

Post-NDE changes include those related to the way the near-death experiencer (NDEr) perceives one's self and tends to focus on one's presumed transcendental nature. Among the most prominent of these changes is loss of fear of death, which has been reported across numerous studies (e.g., Flynn, 1986; Groth-Marnat \& Summers, 1998; Noyes et al., 2009; Ring, 1984; Sabom, 1982; Sartori, 2014; Sutherland, 1990; van Lommel, van Wees, Meyers, \& Elferrich, 2001). In their seminal study, van Lommel and colleagues (2001) found loss of death fear was maintained after the NDE at both 2- and 8-year follow-ups.

In line with such findings, research has indicated a concomitant increase in afterlife beliefs among NDErs (Flynn, 1986; Grey, 1985; Greyson \& Stevenson, 1980; Ring, 1980; Sabom, 1982; Sutherland, 
1990). For example, in comparison to a control group of people who also came close to death but did not report recalling an NDE, NDErs were more likely to report a new or strengthened belief in the afterlife (van Lommel et al., 2001). Lai and colleagues (2007) found $75 \%$ of Taiwanese dialysis patients who had an NDE reported a decreased fear of death, and 56\% reported a belief in 'life after life.' When questioned further about their changed attitude to death, $43 \%$ claimed it was related to a belief in life after life, which some participants referred to as reincarnation.

Post-NDE, experiencers can also report feeling a heightened sense of self-esteem that is often combined with a renewed sense of purpose, implying that they now know what their life path is and that they have a mission to fulfill in life (van Lommel, 2010). This appreciation is also evident as experiencers take more time to savor the moment and really appreciate the small things in life (Sartori, 2014; van Lommel, 2010). A newfound appreciation for life can also be seen in some individuals who experience a distressing NDE. This appreciation manifests as a desire to 'turn one's life around,' especially when one perceives the NDE as a warning with the opportunity for redemption, which may involve invoking religious means to become eligible to enter 'heaven' (Bush, 2002) or through NDErs becoming less judgmental and having an increased love of life as well as heightened morality (Sartori, 2014).

\section{Changes in Relationships to Others}

The aforementioned changes in self-perception have a subsequent effect on interpersonal relationships. For example, many NDErs report a greater sense of compassion and love toward others. This positive affective stance is typically associated with new ways of interacting that include being less competitive, less critical, more forgiving, and more ready, willing, and open in expressing how they feel toward others. Many NDErs report enhanced tolerance as a result of their NDEs (Sartori, 2014). These changes in compassion, love, and tolerance are often immediately noticeable (Groth-Marnat \& Summers, 1998; van Lommel, 2010), resulting in changes to family relationships, as experiencers may spend more time enjoying and appreciating their families.

Although experiencers describe these relationship changes as largely positive, the breakdown of significant relationships and employment dissatisfaction and subsequent change have also been noted (Christian \& Holden, 2012; Groth-Marnat \& Summers, 1998; Musgrave, 1997; Stout, Jacquin, \& Atwater, 2006). NDErs can struggle to 
communicate, due to the ineffable nature of NDEs and experiencers' lack of vocabulary available to describe their feelings. Friends and family can find it difficult to adjust to the 'new' version of their loved one, who appears to have undergone some fundamental changes regarding who they are as a person. Other difficulties may arise from the experiencers' desire to redirect their newfound compassion to help others. This desire may translate into a career change to professions such as nursing or terminal patient care or into volunteer work for the elderly/low income families. NDErs are also more likely to donate to charities or volunteer their services to a particular cause (van Lommel, 2010). These drastic changes in interests and values can be difficult for a spouse or family to adjust to and may lead to divorce (Christian \& Holden, 2012; Sutherland, 1990). Some NDErs may question their own sanity after having had an NDE, which — though unfounded based on empirical research showing no particular relationship between NDEs and mental health-can be further exacerbated if significant others or medical professionals do not respond to the NDEr supportively (Holden, Kinsey, \& Moore, 2014; Morris \& Knafl, 2003).

\section{Changes in Attitudes Toward Life}

NDErs tend to evidence change in their perceptions of life and in how they live. They often report a greater appreciation for nature, living, and knowledge acquisition, along with a renewed zest for life. NDErs can have a dramatic change in interests, in hobbies, and in religious faith or abandonment of religious faith. They can feel "as if I had become a different person but with the same identity" (van Lommel, 2011, p. 22). Both Noyes (1980) and Flynn (1986) suggested the decreased fear of death acts as a precursor to such changes.

A further attitudinal change is a new or heightened sense of spirituality. Some people report a heightened sense of religiosity, whereas others feel that affiliation with a particular religion is no longer beneficial (Sartori, 2014). Some NDErs may become less religious but identify as more spiritual (Sutherland, 1990). More recently, a greater amount of enhanced spiritual well-being (Khanna \& Greyson, 2014a), spiritual growth (Greyson \& Khanna, 2014), and quantity of daily spiritual experiences (Khanna \& Greyson, 2014b) have been reported in those who have had an NDE compared to those who survived a close brush with death with no NDE. 


\section{Present Study}

Evidence for the impact of pleasurable Western NDEs is well established and implies a consistent pattern of post-NDE attitudinal and belief changes evident across cultures. Although Noyes and colleagues (2009) cited a variety of major studies that have produced these results, such studies were predominantly conducted within the United States, United Kingdom, and Australia. Indeed, most of what is currently known about NDEs comes from literature, including case studies and research, conducted in Western Anglo-European cultures (Kellehear, 2009). A substantial minority of NDE cases have been reported in non-Western cultures, such as Hawai'i (e.g., Kellehear, 2001), Thailand (e.g., Murphy, 2001), India (e.g., Pasricha, 1993), South America (e.g., Gómez-Jeria, 1993), and Israel (Abramovitch, 1988). Although useful and beneficial in its own right, the current NDE research field largely reflects the worldviews, values, and perspectives of particular Western cultural groups (Berry, Poortinga, Segall, \& Dasen, 2002). Consequently, much of the academic information generated about NDEs derives from a Western lens or perspective and in many ways is treated as the reference point for NDEs.

The growth of indigenous psychology has been instrumental in raising awareness that alternative realities exist for individuals depending on their cultural-including religious-affiliation and context. Not restricting themselves to indigenous peoples per se, scholars of indigenous psychology have suggested that all psychological theories, methods, and processes are indigenous to the local context in which they were developed (Kim \& Berry, 1993, p. 2). One of the objectives of indigenous psychology is to question the (assumed) universality of current psychological theory and to explore phenomena, methods, and theories from localized perspectives while also determining their congruence with the universalized perspectives that dominate many academic disciplines.

To our knowledge, no studies published in refereed journals have assessed NDE aftereffects in culturally and ethnically diverse Western nations outside of the US and Western Europe, such as Aotearoa New Zealand. The current population of Aotearoa New Zealand is comprised of approximately 14\% indigenous Māori, 74\% of European descent, and 12\% Pacific Peoples, Asian, and other ethnicities (Statistics New Zealand, 2013a). According to Spoonley (2015), the country is considered a 'settler society,' which is defined as one that contains both "national minorities comprised of indigenous peoples who have 
been colonized, and ethnic minorities whose presence and compositional characteristics are the product of both historical and contemporary immigration policies and a nation building project that revolves around immigration" (pp. 651-652). Consequently, settler societies such as Aotearoa New Zealand serve as meeting points for cultural groups and the diverse worldviews that accompany such groups. Exposure to such diversity leads to acculturation and subsequent changing of cultural beliefs, values, practices, and rituals over time (Marin \& Gamba, 2003). Years of "colonisation, exploitation, and oppression" (Walker, Eketone, \& Gibbs, 2006, p. 332) facilitated the erosion of indigenous Māori cultural institutions, including language and traditional ways of being. Despite this change, some cultural traditions have been preserved and are evident in contemporary Māori culture (Dansey, 1992; Jacob, Nikora, \& Ritchie, 2012; Nikora, Masters, \& Te Awekotuku, 2012). These traditions feed into contemporary society, which is comprised of varying ethnicities, creating a unique cultural milieu that distinguishes Aotearoa New Zealand from cultures in other parts of the world.

One cultural dimension of particular relevance to NDE research, but also to notions of cultural diversity, is religious or spiritual affiliation. In Aotearoa New Zealand, the proportion of people who affiliate with any of the major Christian religions continues to fall and is now less than half of the country's population (48.9\%), whereas the proportion indicating 'no religion' continues to increase and now constitutes $41.9 \%$ of the country's population. Younger people, males, and those identifying with the Māori and European ethnic groups are most likely to report they have no religious affiliation. The proportion of people reporting they affiliate with religious traditions other than Christianity-including Muslim, Hindu, Buddhist, and Sikhcontinues to increase, which may be related to the 'settler' society' demographic of the country (Statistics New Zealand, 2013b). The continued decline of Christian-based religions, increase in non-Christianbased religions, and increase in those not adhering to any religious philosophy has contributed to descriptions of Aotearoa New Zealand as a "secular" society (Pratt, 2016).

Exploring the cultural diversity of NDEs was recently highlighted as a research priority (e.g., Sleutjes, Moreira-Almeida, \& Greyson, 2014). Consequently, research with the unique population of Aotearoa New Zealand promises to enrich the knowledge base regarding NDEs and their aftereffects by applying an indigenous psychological philosophy to examining NDEs. Following on previous research examining 
NDE phenomenology with samples from Aotearoa New Zealand (e.g., Tassell-Matamua, 2013; Tassell-Matamua \& Murray, 2014), the purposes of the present study were to examine how NDErs from within an Aotearoa New Zealand context described any post-NDE changes they had experienced; how their descriptions compared to those from previous research conducted in other cultural contexts such as the US; and, if notable differences were found, whether such differences might be explained in terms of cultural context.

\section{Method}

Participants. Initially, 30 individuals who self-identified as having had an NDE expressed interest in participating in an interview about their NDEs. When contacted, 15 maintained their interest in participating, although only six were able to travel to the interview venue, of which only four were able to travel during the specified research time period. These four participants - two female and two male, ranging in age from 47-70 years old-were interviewed about their NDEs. Participant occupations included work in politics, part-time work in customer service, and work as a researcher; one participant was retired. All four participants identified as Pākehā-a common term used in Aotearoa New Zealand to describe those born in Aotearoa New Zealand of European descent. No other demographic details were obtained.

Debates about sample size in qualitative research continue, with no clear guidelines on the number of participants necessary to obtain 'enough' data. Rather, issues such as the type of qualitative analysis, access to participants, and saturation are important considerations when considering how many participants to interview. Given we were interested in the phenomenology of participant accounts of NDE aftereffects, we decided to use the guidelines provided by Cresswell (1998) and Morse (1994), which indicate that 5-25 participants are sufficient for phenomenological studies. When we were unable to obtain at least 5 participants to interview, we decided to include written transcriptions in the analyses. Co-authors Tassell-Matamua and Steadman had regularly received unsolicited accounts of NDEs from NDErs directly emailing them. Some of these accounts were from individuals either who were unable to attend an interview-most due to geographical distance-or who wished to remain anonymous for research purposes. The authors responded to a total of 28 individuals who sent unsolicited NDE accounts during the period the research was under- 
taken, providing them with the interview questions and inviting them to submit a written account that addressed those questions. Our initial expectation was that half might choose to participate. However, during the research time period, 24 of these 28 individuals addressed the interview questions in written accounts they returned via email or postal mail. Demographic details about these participants are not known, given we did not specifically ask for those data to be included in the written submissions.

Demographic information is often useful for comparative purposes and to provide a more detailed picture of NDErs. However, we were interested in the themes and sub-themes inherent to participant accounts, for the purposes of exploring potential differences across cultures as opposed to within cultures. That is, we were interested in looking at whether descriptions of change from NDErs in Aotearoa New Zealand might differ from descriptions and identified changes reported in previous literature with Western samples. We were also not interested in comparing NDE aftereffects according to specific demographic factors beyond those assumed by country- or nation-level culture. Given some participants did not wish to be interviewed but rather wished to submit a written account, we also felt compelled to respect those wishes and not re-contact participants who had provided a written narrative to request additional information, such as demographics. We understand the omission of demographic information may be considered a limitation of the study but believe the rationale for the omission is theoretically and pragmatically justified.

Procedure. Recruitment occurred via a snowballing method, using the acquaintanceship networks of the first two authors, the first of whom is an experienced researcher of nine years in the field of neardeath studies and has herself had an NDE. Although researchers in quantitative studies of NDEs typically administer the Near-Death Experience Scale (NDE Scale; Greyson, 1983) as a means to verify whether the experience meets objective criteria for an NDE, we chose not to administer the scale. Given that NDErs have reportedly felt harmed when their disclosures were met with disbelief (Holden et al., 2014; Wilde \& Murray, 2009), we did not wish to give the perception of minimizing any individual experiences presented to us by assessing such experiences against the specific criteria in the NDE Scale. Instead, we accepted participants' self-identification as having had an NDE. Written accounts and interviews involved participants describing their NDEs, and prior to finally including participants in the study, Tassell-Matamua and Steadman assessed these descriptions to 
determine that they contained enough detail and typical characteristics to classify them as NDEs. We concurred unanimously that all submitted written accounts met this standard.

Data collection involved conducting semi-structured interviews (see Appendix for interview schedule) and receiving written responses to the same interview questions. Interviews were conducted in the educational institution where the research was hosted, lasted approximately one hour, and followed a semi-structured format involving the interview schedule's fixed prompts and then, if appropriate, individualized follow-up questions. Written responses to the fixed prompts were provided via postal mail or email.

For both interviews and written submissions, participants described their NDEs in detail and were specifically asked to note any changes they experienced as a result of their NDEs. Two participants indicated they had experienced two NDEs, whereas another indicated he had experienced the same NDE (with the same features) three times on the same night. All of these participants had provided written responses, and none of them separated out the effects of their respective NDEs, making it difficult to ascertain if specific aftereffects related to one or more of the specific NDEs described. Once completed, interviews were transcribed. Both the interview transcripts and written accounts pertaining to post-NDE changes were thematically analyzed using the protocol outlined by Braun and Clarke (2006), which is "a method of identifying, analyzing and reporting patterns (themes) within data" (p. 79). This process involved six phases:

(a) Familiarization with the data by Steadman;

(b) An initial coding process before identifying broad codes within the explicit content of the data by Steadman;

(c) Categorizing of themes across the data set based on the identified initial codes by Steadman;

(d) A review of the identified themes to ensure they corresponded with the identified codes by Steadman and Tassell-Matamua;

(e) Defining, naming, and refinement of the themes and any subthemes by Steadman and Tassell-Matamua; and,

(f) Writing up of the final themes and sub-themes with appropriate quotes to illustrate each.

\section{Results}

We chose to name each of the major themes using the three categories highlighted by Noyes and colleagues (2009) - perception of self, relationships to others, and attitudes toward life-as these corresponded readily with many of the changes reported in previous literature and 
Table 1 Total Number of Participant Narratives Referencing Specific SubThemes $(\mathrm{N}=28)$

\begin{tabular}{llccc}
\hline \multicolumn{1}{c}{ Main theme } & \multicolumn{1}{c}{ Sub-theme } & $\begin{array}{c}\text { Evident } \\
\text { in written } \\
\text { responses }\end{array}$ & $\begin{array}{c}\text { Evident in } \\
\text { interviews }\end{array}$ & Total \\
\hline Perception of self & $\begin{array}{l}\text { Loss of the fear of death } \\
\text { Beliefs in an afterlife }\end{array}$ & 19 & 3 & 22 \\
\hline Relationships to & Disclosure of NDE & 11 & 3 & 10 \\
others & Changed relationships & 10 & 3 & 14 \\
& Tolerance of others & 8 & 1 & 12 \\
& Others noticing changes & 3 & 3 & 6 \\
\hline Attitudes toward & Enhanced spirituality & 10 & 2 & 12 \\
life & Changed religious & 6 & 4 & 10 \\
& perspective & & & \\
\hline
\end{tabular}

provided a useful point of reference for making comparisons between the changes identified by our sample and changes previously identified in other studies. We also identified sub-themes within these three major themes. Table 1 provides an overview of how many participants described each of the specific sub-themes organized by most to least frequently referenced in participant narratives. The following text provides excerpts from participant narratives that exemplify each sub-theme.

Changes in perception of self. Changes in perceptions of self tended to reflect psychological changes in attitudes, values, perceptions, and worldviews, as well as a new or renewed sense of one's transcendental nature. Participants described changes they had noted in themselves before indicating whether or not they accepted these changes. Identified sub-themes included loss of the fear of death and beliefs in an afterlife.

Loss of the fear of death. Perhaps unsurprisingly, the most notable change in perception of self was a loss of the fear of death. A total of $79 \%$ of participants explicitly expressed this sub-theme. Although some participants indicated apprehension about how they might die, many described no longer fearing death or holding previously held beliefs about the finality of death: 
No, I had a fear of death before I was a Christian; I used to be really fearful about it. I thought it was so final, because of the way we were brought up. So I didn't realise until it was gone how great it was not to have that. So no, I don't fear it at all. (Participant B)

What I had afterwards, was having the knowledge of never fearing death in the future! (Participant 1)

. . all I got was a glimpse, as it were, of a very beautiful place; and a very real sense that we do not need to fear the passing into the next life. (Participant 5)

I have had an overwhelming sense of peace and calm and certainty about life in a different parallel Universe. Ever since, I am not afraid of death. (Participant 10)

My near-death experience has never left me . . . I had no idea what was happening, but remember it very clearly. What it did do was relieve me of my fear of death. (Participant 13)

I, for one, am not afraid of death and am only excited about what is next once I have left my physical body behind. (Participant 21)

Beliefs in an afterlife. In line with changes in attitudes toward death, $35 \%$ of participants expressed that their beliefs about the afterlife had also been transformed. Many indicated a "not knowing" before their NDE but confirmed they now accepted there is an afterlife:

Before I was like "I wonder if," whereas now I am inclined to believe there is. If you had asked me before I would have said maybe, whereas now I am more leaning toward yes there is an afterlife. (Participant A)

Yeah, I expect an eternal life when I die. My spirit and soul to go on. I expect that it will just be the communication but at a deeper level ... I think the body disintegrates. But, I think the breath of life. I think when your breath goes, you leave your body, the core or essence or spirit is alive... (Participant B)

The irony is that it's not a death at all, just a spontaneous transition as easy and natural as breathing in ... My NDE can confirm one fact known to me. This so-called 'heaven'-place of the life-there-after-is not in an upwards direction. It's all around us. The NDE revealed that the transformation from the physical world to that other one is like changing channels, or lifting a veil. (Participant 8)

Changes in relationships to others. Changes in relationships with others included descriptors reflecting acceptance of others. Also noted were positive changes significant others had noted about the NDErs, which influenced their subsequent relationship with them. Partici- 
pants also spoke about disclosure of the NDE as both positively and negatively influencing interpersonal interactions. Sub-themes identified included tolerance of others, changed interpersonal relationships, others noticing changes, and disclosure of NDE.

Tolerance of others. A total of $32 \%$ of participants reflected that their new outlook on themselves enabled them to be more 'open-minded' and 'tolerant of others' perspectives.'

Up until my NDE I was open-minded about a lot of things, but very much a person who had my feet in the world of logic and science, with some open-mindedness toward the unexplained. Post NDE, I feel as if I have my feet in both worlds, and the two worlds are surprisingly compatible. And, I've got a very strong sense that I'm very much part of a much bigger whole, and to just go along with the synchronicities that have played a large part in my life. (Participant 17)

You know you had your ideals and you'd be discussing them with people and if they didn't agree, you would try and explain to them how you felt. Now it's different, and hey people have their views and I wouldn't consider trying to change it. You have a discussion and I respect their views and my views. And so be it. (Participant B)

My NDE does bring me a rock-solid, unshakeable calm when helping others with loss, crisis and trauma. Maybe somehow, in some way that inner peace translates to the people I'm helping. (Participant 8)

My life since my NDE has changed considerably. I have become a better person. More understanding, compassionate, caring, relaxed, and less opinionated. (Participant 6)

I think I became more laid back as a result of all of this. (Participant 20)

Changed interpersonal relationships. This tolerance of and openness to others perspectives had implications for interpersonal relationships and interactions. Many participants (43\%) felt greater acceptance of and connection with others while also valuing social institutions such as family.

I found that a purpose in this life has more to do with connecting with people and enhancing their wellbeing. Relationships matter and it's what you do for people is what counts more than what you do for yourself. Ask anyone on their death-bed regards what really mattered to them in their life journey, and nearly always it results in their answer involving 'people,' not how much money they made or how many empires they built. (Participant 8) 
It made me value life, it made me quite cautious . . . It really made me take account of my life, my health, and my family. (Participant D)

What I experienced will never leave me, but I can also tell you I'm here to help people for some reason. (Participant 23)

The importance placed on interpersonal relationships was also expressed by one participant as a need not to waste time engaging with people who were not of certain qualities while also accepting that other people are not required for one's own sense of worth:

I don't waste time with people who are negative or vindictive or cynical, am more assured about my own opinions, and don't need the approval of others to make it through life. This world is truly a beautiful place, something I had not noticed before this crisis. (Participant 10)

Others noticing changes. In addition to describing the positive changes to their own approach to interpersonal relationships, $21 \%$ of participants indicated significant others had noted these changes. The most acceptance was noted from family members, although some participants indicated friends also acknowledged the changes.

I believe those around me can see I have changed for the better, many have said so. My sister is always saying that she likes the new me better. I am more content now and not so driven. I still want and desire a good life, but I want and desire happiness and contentment more. (Participant 6)

Some of them now think I am a bit more laid back. I have always tried to help people. I volunteer. I still do that. I suppose if anything people think I am a bit more laid back. It is just that I just take it as it goes. (Participant A)

Disclosure of NDE. Despite the positive reactions that many participants expressed, some did not experience acceptance of their changes. A total of $50 \%$ of participants indicated they had not disclosed their NDE to anyone, as they were fearful of the lack of acceptance that might result, through being ridiculed or labeled "silly" or "crazy."

I have been telling no one about it because people will call me silly. Even I have not told my children about it to date. I am sharing it with you guys for the first time. (Participant 26)

I never found it easy to talk about this experience, as I believed people would think I'm crazy. (Participant 21)

I felt weird and confused for ages after it and told no one, not even the ambulance people who attended me. (Participant 27) 
Of those that had disclosed their NDE, the reactions of others were often mixed. Mostly others could not accept the psychological changes that had occurred:

... Yeah, people always surprise me. It is quite sad. It was quite hard working through ... living with it. It does surprise me when people act in such an immature and selfish way. So their expectations and my husband's expectations became unrealistic of me. (Participant B)

My husband couldn't comprehend my reasons for change. I have found it impossible to share it with any family at all, which has rather alienated aspects of family life. (Participant 22)

I do not think any of my family was too impressed with my new life and generally did not share my opinions, so I learned to keep my beliefs to myself ... My friends were not too keen to see me. (Participant 4)

When I told people about my experience they listened politely, but apart from my husband no one really asked questions at all. I told the Catholic priest and he didn't say much at all-just smiled politely! (Participant 11)

Medical personnel were mostly dismissive of the NDE, although one participant noted a positive, 'sympathetic' reaction from a general practitioner physician (GP):

I talked to a few and they said it was interesting. I didn't push it and they didn't ask, so it didn't go any further. You see it often, a lot of doctors don't even believe in natural medicine. (Participant A)

I told my mum about it, who didn't believe me. When we went for the check-up she asked the doctor if this could have been possible. They got me to tell the surgeon what I had heard, and he just looked at me shocked and walked away. (Participant 19)

I have told my GP about this and he was most sympathetic and made me feel believed. (Participant 3)

Changes in attitudes toward life. Participants typically indicated their attitude toward life had changed in varied ways since their NDEs. Sub-themes identified were changes in religious perspectives and an enhanced sense of spirituality.

Changes in religious perspectives. These changes were especially prominent, with $35 \%$ of participants expressing this sub-theme. Changes tended to vary between strengthening or refuting existing religious beliefs. 
I think after it has just strengthened my faith and strengthened my ... it has just been one of the things . . . several things have happened to me. It is just another thing that has laid a foundation. (Participant B)

I already had been strong in my Christian faith and believed in life after death, but this experience only strengthened that ... (Participant 14)

I have always had my faith, I don't go to Church as I don't believe in it, but I have always believed in God. I keep this to myself and inside me. I know more than ever now that God exists, I also believe in Guardian Angels. I know they saved me. (Participant 6)

Other participants, however, indicated their NDEs provided them with a different perspective on religion-one in which they perceived religion to be a "manmade" construct, with negative connotations that participants no longer required:

I also had been well drilled in religion; I found the whole thing repugnant. So, I was wrestling with the duality of religion on one side and spirituality to one side. I could not integrate ... I have come to the point where I see clearly what religion is about. I see a sort of negative consciousness that has been imposing its view on us. (Participant C)

The outcome was the realisation that religion is a manmade construct that crudely attempts to make sense of life and what may lie beyond that. Often these [are] driven by fear and coercion, in which each religion thinks it is the exclusively correct one and all other believers contrary to their own, must confront some vague catastrophic doom for being different. (Participant 8)

I was brought up in a very religious household but no longer need that "prop," and firmly believe I will be reunited with those who have gone when my time comes. (Participant 10)

... it was explained [to me during my NDE] that "God," spirits, religion are just perception filters used when contact was made with "them"... (Participant 16)

Enhanced sense of spirituality. Some participants indicated their NDE resulted in a personal journey of exploration and discovery, which resulted in a greater acceptance of one's sense of spirituality. Just under half of all participants (43\%) expressed this sub-theme.

My personal journey of seeking the closest explanation for what my NDE meant resulted in the study of several mainstream and eastern religions. I'm still not religious, never was, and definitely never will be. There is a 'god,' some higher energy on the scale of things. That I know. (Participant 8) 
Well, I think as far as that . . I think it has reassured me spiritually. (Participant B)

As a result, I had a massive spiritual experience which was somewhat disconcerting to this self-professed atheist. But, it made me rethink my stance about God, and the meaning of life, and especially my place in the Universe. (Participant 4)

\section{Discussion}

The primary purpose of our study was to examine how NDErs in Aotearoa New Zealand described any changes they had experienced after their NDEs. Overall, our data suggests NDErs from Aotearoa New Zealand identified similar changes to those of NDErs described in previous literature that stems primarily from Western cultures. However, findings also highlighted some discrepancies from previous findings.

\section{Perceptions of Self}

Changes in attitudes towards death, which included a greater acceptance of death and the existence of an 'afterlife'-or life beyond the bounds of the current physical life-were described. One participant also spoke of coming to an understanding about a purpose in life. Participants typically spoke about not endorsing such perspectives prior to their NDEs but certainly feeling comfortable with changes in perspective after their NDEs. Such changes support those recorded in a host of other NDE research (e.g., Flynn, 1986; Groth-Marnat \& Summers, 1998; Lai et al., 2007; Noyes, 1980; Sartori, 2014; van Lommel et al., 2001). However, other changes to perception of self that previous researchers had noted (e.g., Noyes et al., 2009), such as a heightened sense of self-esteem, were not described by our sample. This finding does not mean such changes did not occur for our participants, but they certainly were not explicitly described. An explanation for this result may be the Tall Poppy Syndrome (TPS), which has been identified as a culturally-specific phenomenon that perpetuates a social norm of not bragging or boasting about oneself and one's achievements, positive qualities, aims, and ambitions (Feather, 1991; Kirkwood, 2007; Pierce, Hodge, Taylor, \& Button, 2017). TPS is characteristic of former colonial countries or 'settler' societies, such as Aotearoa New Zealand and Australia, with much research supporting its existence in these cultural contexts (e.g., Holmes, Marra, \& Lazzaro-Salazar, 
2017; Peeters, 2004). Given the prevailing nature of the TPS in Aotearoa New Zealand culture, the lack of descriptions of heightened selfesteem by NDErs in our sample, seems congruent.

Previous literature typically defined physical aftereffects as an altered perception of the body, which can include a sense of being transformed, feeling alienated from the physical body but somehow still a part of it, and/or experiencing physiological aftereffects such as electromagnetic phenomena (e.g., Atwater, 1988; Foster, James, \& Holden, 2009; Greyson, Liester, Kinsey, Alsum, \& Fox, 2015). Physiological effects were not discussed in detail by participants, although this absence does not suggest the sample did not experience them. In contemporary Western cultures, conventional understandings of normative reality are based on a material reductionist philosophy emphasizing established scientific principles and laws, such as cause-and-effect, objectivity, positivism, and empirical verification (Fach, Atmanspacher, Landolt, Wyss, \& Rossler, 2013; Landolt, Wittwer, Wyss, Unterassner, Fach, et al., 2014). Much skepticism surrounds any human experiences contravening these understandings, such as the electromagnetic phenomena typically described by some NDErs, which feeds into a social norm of not speaking about or openly endorsing such phenomena as probable or even possible. Given the relatively secular nature of Aotearoa New Zealand culture (Pratt, 2016), such scientific predispositions to explaining normative reality and phenomena, rather than religious and/or spiritual, are likely to be prevalent. Although participants chose to disclose their NDEs, which are phenomena that inherently contravene established understandings of reality, participants may not have been comfortable disclosing physiological aftereffects because the secular nature of Aotearoa New Zealand does not readily provide an explanatory framework for why such effects should occur after an NDE.

Of note, some participants in our sample did discuss physical changes that had typically resulted from incidents that were the catalyst for the NDE or were an aftereffect of the initial physical injury associated with the NDE, rather than being changes directly attributable to the NDE itself. Physical changes were discussed as problematic and generative of unpleasant and/or distressing psychological effects. In Aotearoa New Zealand, dominant societal discourses typically equate healthy lifestyles with being physically active and able (Markula 1997), which implicitly suggest that any physical impairment may equate to less potential to lead a healthy lifestyle. It may be that our sample, in comparison to previous samples, comprised a 
higher proportion of individuals who had their NDEs in association with some traumatic physical incident, and the dominant societal discourses mentioned above resulted in participants describing their physical changes as problematic. Their problematic nature and association to the NDE may have led some participants to perceive and talk about the physical changes as an NDE aftereffect, although in fact they were an aftereffect or consequence of the traumatic physical event.

\section{Relationships with Others}

NDErs' transformations in self-perceptions often catalyzed changed relationships with others. Participants explicitly described general changes to the way they perceived other people, which involved greater tolerance and acceptance, irrespective of who the person is and whether that person's opinions, perspectives, and worldviews are similar to or different from those held by the NDEr. In her recent work, Penny Sartori (2014) also noted similar changes in levels of tolerance towards others, thus supporting the present findings. Our participants noted the changed dynamics they felt in interpersonal relationships but also acknowledged significant others had noticed changed interactions with them. Although others were often encouraged by these interactions, they were not always comfortable with the reasons for the change- that is, the NDE. Previous research indicates the breakdown of significant relationships and even divorce may be higher among those who have NDEs, in comparison to the general population (Christian \& Holden, 2012). Whereas our sample tended to describe positive changes in interactions with family and friends, one participant did describe difficulties with her husband comprehending the changes she had made post-NDE, and another described difficulties with family and friends accepting his/her new post-NDE beliefs. It is not known whether such difficulties eventually led to the dissolution of those relationships, but the difficulties experienced align to some degree with previous findings.

Although one participant mentioned that money and empires were unimportant in life, overall explicit expressions related to a lessened concern for material gain, status, and/or recognition noted in previous literature (e.g., Noyes et al., 2009) were not evident in the descriptions of our sample. Given the assumed prevalence of TPS within the Aotearoa New Zealand cultural context (e.g., Holmes et al., 2017; Peeters, 2004), status and recognition are unlikely to be overtly stated by individuals in the general population, let alone by individuals who have 
had an NDE, as such expressions could be frowned upon as boastful or bragging. The lack of a lessened concern for these issues by our sample is potentially attributable to them not being as openly prized in the Aotearoa New Zealand cultural context as they might be in other Western cultures. Therefore, materialistic values did not dominate the perspectives of our participants and were not identified as notable changes.

Non-disclosure to others of the NDE has often been attributed to social determinants such as a fear of ridicule or disbelief (Duffy \& Olson, 2007; Holden et al., 2014). Our findings support this dynamic, with some participants indicating they had not previously disclosed their NDE to anyone due to fear of being labeled 'crazy' and/or not being believed. Participants who did disclose their NDE to others described mixed reactions from their confidants. Notably, medical professionals did not always respond well to NDE disclosures, which is in line with other findings suggestive of the same reaction. For example, disclosure of NDEs to health professionals has not always been received favorably (Greyson, 1997), with some professionals pathologizing the experience as a hallucination, reducing it to impaired neurophysiology, or dismissing it as inconsequential (Duffy \& Olson, 2007). More recently, Holden and colleagues (2014) found those who report more intense NDEs and/or disclose their NDE shortly after the experience were most likely to report reactions from health professionals that the NDErs described as harmful to them. Our findings continue to highlight a concern with regard to NDE disclosure reactions by professionals within health settings, irrespective of cultural context, and support a general trend of perceived stigma as a main causal factor for individuals avoiding or delaying disclosure of certain experiences to health professionals (Clement et al., 2015).

\section{Changes in Attitudes Toward Life}

Changes in attitudes toward life did not manifest as a greater appreciation for nature, living, knowledge acquisition, and a renewed zest for life, as previous researchers had reported (e.g., Noyes et al., 2009). However, once again this finding does not necessarily indicate these changes did not occur for our sample but, instead, suggest they may not have been as pertinent or within conscious awareness when participants were considering the changes that had occurred for them post-NDE.

Instead, the primary foci for attitude change centered on religion 
and spirituality. As a secular society, recourse is not given to religion and spirituality when it comes to political and social concerns in Aotearoa New Zealand (Pratt, 2016). Nevertheless, some participants reported their post-NDE religious beliefs and affiliations had strengthened, whereas others expressed a heightened conviction that religion is more associated with orthodoxy and doctrine and/or is socially/ culturally constructed. Additionally, participants were able to differentiate between changes that were religiously oriented and those more spiritually oriented, with many indicating an increased sense of 'spiritually' as a primary outcome of their NDE. Although such findings align with previous research highlighting the differentiation made between an enhanced sense of spirituality, as opposed to an enhanced sense of religiousness, post-NDE (e.g., Sutherland, 1990; Musgrave, 1997, Tassell-Matamua \& Steadman, 2017), they also point to the possibility that the relative secularity of a particular culture may affect post-NDE notions of religiosity and spirituality in divergent ways.

\section{Limitations of the Study and Future Research}

Considering that the purpose of this study was to compare post-NDE changes between previous samples of predominantly Western cultures and a sample from the culturally unique and diverse population of Aotearoa New Zealand, the absence of demographic data is an important limitation. In particular, it cannot be known the extent to which our sample represented the cultural diversity of Aotearoa New Zealand. For example, it is possible that all of our participants came from the $74 \%$ of people of European descent, which would account for the similarity between our findings and those of studies of Western cultures. Future researchers are encouraged to request the simple demographic data of age, sex, and ethnic affiliation. However, it is equally important to emphasize that as a cross-cultural study investigating differences across rather than within cultures, there is an inherent understanding that this study was exploring any variations in aftereffects reported by NDErs living in the Aotearoa New Zealand cultural context versus those living in other cultural contexts where NDE research has been reported, such as the US. As has previously been described, the cultural context of Aotearoa New Zealand is unique and differentiated from other Western nations around the globe, so individual-level demographics become subsumed within the context of the larger cultural milieu. 
Despite these limitations, this exploratory study yielded more than enough data to warrant further research on this topic. Future qualitative researchers might ask more pointed questions regarding the themes that Noyes et al. (2009) identified. Again, weighing the costs and benefits of various methodological approaches, quantitative inquiry might yield helpful data in understanding post-NDE changes and their culturally associated factors.

\section{Conclusion}

Although major studies consistently report evidence of a pattern of specific changes that occur after an NDE, no studies to date have assessed whether such changes are evident in culturally distinct samples such as Aotearoa New Zealand, how any changes compare to those described in existing research, and whether differences might be explained by culturally-specific considerations. Findings from our exploratory qualitative study suggest many of the changes reported by a sample from the Aotearoa New Zealand cultural context are not that dissimilar to those previously reported by other NDErs, at least in Western nations. However, some discrepancies were evident, which we have indicated may be related to unique factors of the socio-cultural milieu of Aotearoa New Zealand. Our findings suggest cultural context may be an essential element to consider when examining both how and what aftereffects are described by individuals after an NDE. They also highlight the importance of NDE research being done in other cultural contexts beyond those typically considered 'Western' to expand the pool of knowledge about NDEs, and we encourage future investigations of this nature to be undertaken.

\section{References}

Abramovitch, H. (1988). An Israeli account of a near-death experience: A case study of cultural dissonance. Journal of Near-Death Studies, 6, 175-184. doi:10.17514/JNDS-1988-6-3-p175-184.

Atwater, P. M. H. (1988). Coming back to life: The after-effects of the near-death experience (rev. ed.). New York, NY: Citadel.

Atwater, P. M. H. (2009). Beyond the light. What isn't being said about neardeath experiences (rev. ed.). Kill Devil Hills, NC: Transpersonal.

Berry, J. W., Poortinga, Y. H., Segall, M. H., \& Dasen, P. R. (2002). Cross-cultural psychology. Research and Applications (2nd ed.). Cambridge, England, UK: Cambridge University Press.

Braun, V., \& Clarke, V. (2006). Using thematic analysis in psychology. Qualitative Research in Psychology, 3, 77-101. 
Bush, N. E. (2002). Afterward: Making meaning after a frightening near-death experience. Journal of Near-Death Studies, 21, 99-133. doi:10.17514/JNDS2002-21-2-p99-133.

Christian, R., \& Holden, J. M. (2012). 'Til death do us part': Marital aftermath of one spouse's near-death experience. Journal of Near-Death Studies, 30, 207231. doi:10.17514/JNDS-2012-30-4-p207-231.

Clement, S., Schauman, O., Graham, T., Maggioni, F., Evans-Lacko, S., Bezborodovs, N . . Thornicroft, G. (2015). What is the impact of mental health-related stigma on help-seeking? A systematic review of quantitative and qualitative studies. Psychological Medicine, 45, 11-27.

Creswell, J. W. (1998). Qualitative inquiry and research design: Choosing among five traditions. Thousand Oaks, CA: Sage.

Dansey, H. (1992). A view of death. In M. King (Ed.), Te Ao Hurihuri. Aspects of Māoritanga (pp. 105-116). Auckland, New Zealand: Reed.

Duffy, N., \& Olson, M. (2007). Supporting a patient after a near-death experience. Nursing2007, 37(4), 46-48.

Fach, W., Atmanspacher, H., Landolt, K., Wyss, T., \& Rossler, W. (2013). A comparative study of exceptional experiences of clients seeking advice and of subjects in an ordinary population. Frontiers in Psychology, 4 (Article 65), 1-9.

Feather, N. T. (1991). Attitudes towards the high achiever: Effects of perceiver's own level of competence. Australian Journal of Psychology, 43, 121-124.

Flynn, C. P. (1986). After the beyond. Human transformation and the near-death experience. Englewood Cliffs, NJ: Prentice-Hall.

Foster, R. D., James, D., \& Holden, J. M. (2009). Practical applications of research on near-death experiences. In J.M. Holden, B. Greyson, \& D. James (Eds.), The handbook of near-death experiences. Thirty years of investigation (pp. 235-258). Santa Barbara, CA: Praeger/ABC-CLIO.

Gómez-Jeria, J. S. (1993). A near-death experience among the Mapuche people. Journal of Near-Death Studies, 11, 219-222. doi:10.17514/JNDS-1993-11-4p219-222.

Grey, M. (1985). Return from death: An exploration of the near-death experience. London, England, UK: Arkana.

Greyson, B. (1983). The near-death experience scale. Journal of Nervous and Mental Disease, 171(6), 369-375.

Greyson, B. (1997). The near-death experience as a focus of clinical attention. Journal of Nervous and Mental Disease, 185, 327-334.

Greyson, B., \& Khanna, S. (2014). Spiritual transformation after near-death experiences. Spirituality in Clinical Practice, 1(1), 43-55.

Greyson, B., Liester, M. B., Kinsey, L., Alsum, S., \& Fox, G. (2015). Electromagnetic phenomena reported by near-death experiencers. Journal of Near-Death Studies, 33, 213-243. doi:10.17514/JNDS-2015-33-4-p213-243.

Greyson, B., \& Stevenson, I. (1980). The phenomenology of near death experiences. American Journal of Psychiatry, 137, 1193-1196.

Groth-Marnat, G., \& Summers, R. (1998). Altered beliefs, attitudes, and behaviours following near-death experiences. Journal of Humanistic Psychology, 38, $110-125$.

Holden, J. M., Kinsey, L., \& Moore, T. R. (2014). Disclosing near-death experiences to professional healthcare providers and nonprofessionals. Spirituality in Clinical Practice, 1(4), 278-287. 
Holmes, J., Marra, M., \& Lazzaro-Salazar, M. (2017). Negotiating the tall poppy syndrome in New Zealand workplaces: Women leaders managing the challenge. Gender \& Language, 11(1), 1-29.

Jacob, J., Nikora, L. W., \& Ritchie, J. (2012). Māori children and death: Views from parents. Australian Community Psychologist, 24, 118-128.

Kellehear, A. (2001). An Hawaiian near-death experience. Journal of NearDeath Studies, 20, 31-35. doi:10.17514/JNDS-2001-20-1-p31-35.

Khanna, S., \& Greyson, B. (2014a). Near-death experiences and spiritual wellbeing. Journal of Religion and Health, 53(6), 1605-1615.

Khanna, S., \& Greyson, B. (2014b). Daily spiritual experiences before and after near-death experiences. Psychology of Religion and Spirituality, 6(4), 302-309.

Kim, U. E., \& Berry, J. W. (1993). Indigenous psychologies: Research and experience in cultural context. Newbury Park, CA: Sage.

Kirkwood, J. (2007). Tall poppy syndrome: Implications for entrepreneurship in New Zealand. Journal of Management and Organization, 13, 366-382.

Lai, C. F., Kao, T. W., Wu, M. S., Chiang, S. S., Chang, C. H., Lu, C. S., . . Chen, W. Y. (2007). Impact of near-death experiences on dialysis patients: A multicenter collaborative study. American Journal of Kidney Diseases, 50, 124-132.

Landolt, K., Wittwer, A., Wyss, T., Unterassner, L., Fach, W., Krummenacher, P., . . . Rössler, W. (2014). Help-seeking in people with exceptional experiences: Results from a general population sample. Frontiers in Public Health, 2 (Article 51), 1-9.

Marín, G., \& Gamba, R. J. (2003). Acculturation and changes in cultural values. In K. M. Chun, P. Balls Organista, \& G. Marín (Eds.), Acculturation: Advances in theory, measurement, and applied research (pp. 83-93). Washington, DC: American Psychological Association.

Markula, P. (1997). Are fit people healthy? Health, exercise, active living and the body in fitness discourse. Waikato Journal of Education, 3, 21-39.

Moody, R (1975). Life after life. New York, NY: Bantam.

Morris, L. L., \& Knafl, K. (2003). The nature and meaning of the near-death experience for patients and critical care nurses. Journal of Near-Death Studies, 21, 139-167. doi:10.17514/JNDS-2003-21-3-p139-167.

Morse, J. M. (1994). Designing funded qualitative research. In N. K. Denizin \& Y. S. Lincoln (Eds.), Handbook of qualitative research (2nd ed.). Thousand Oaks, CA: Sage.

Murphy, T. (2001). Near-death experiences in Thailand. Journal of Near-Death Studies, 19, 161-178. doi:10.17514/JNDS-2001-19-3-p161-178.

Musgrave, C. (1997). The near-death experience: A study of spiritual transformation. Journal of Near-Death Studies, 15, 187-201. doi:10.17514/JNDS-199715-3-p187-201.

Nikora, L. W., Masters, B., \& Te Awekotuku, N. (2012). Final arrangements following death: Māori indigenous decision making and tangi. Journal of Community and Applied Social Psychology, 22, 400-413.

Noyes, R. (1980). Attitude change following near-death experiences. Psychiatry, $43,234-242$.

Noyes, R., Fenwick, P., Holden, J. M., \& Christian, S. R. (2009). Aftereffects of pleasurable Western adult near-death experiences. In J. M. Holden, B. Greyson, \& D. James (Eds.), The handbook of near-death experiences. Thirty years of investigation (pp. 41-62). Santa Barbara, CA: Praeger/ABC-CLIO. 
Pasricha, S. (1993). A systematic survey of near-death experiences in South India. Journal of Scientific Exploration, 7, 161-171.

Peeters, B. (2004). Thou shalt not be a tall poppy": Describing an Australian communicative (and behavioral) norm. Intercultural Pragmatics, 1(1), 71-92.

Pierce, S., Hodge, K., Taylor, M., \& Button, A. (2017). Tall poppy syndrome: Perceptions and experiences of elite New Zealand athletes. International Journal of Sport and Exercise Psychology, 15(4), 351-369.

Pratt, D. (2016). Secular New Zealand and religious diversity: From cultural evolution to societal affirmation. Social Inclusion, 4(2), 52-64.

Ring, K. (1980). Life at death: A scientific investigation of the near-death experience. New York, NY: Coward, McCann \& Geoghegan.

Ring, K. (1984). Heading toward omega: In search of the meaning of the neardeath experience. New York, NY: Coward, McCann \& Geohegan.

Sabom, M. B. (1982). Recollections of death: A medical investigation. New York, NY: Harper and Row.

Sartori, P. (2014). The wisdom of near-death experiences: How understanding NDEs can help us live more fully. London, England, UK: Watkins.

Sleutjes, A., Moreira-Almeida, A., \& Greyson, B. (2014). Almost 40 years investigating near-death experiences: An overview of mainstream scientific journals. Journal of Nervous and Mental Disease, 202(11), 833-836.

Spoonley, P. (2015). New diversity, old anxieties in New Zealand: The complex identity politics and engagement of a settler society. Ethnic and Racial Studies, 38(4), 650-661.

Statistics New Zealand. (2013a). Māori population grows and more live longer. Retrieved from http://www.stats.govt.nz/tools_and_services/services/mediacentre/additional-releases/maori-population-estimates-15-nov-2012.aspx.

Statistics New Zealand. (2013b). Religious affiliation. Retrieved from http:// archive.stats.govt.nz/Census/2013-census/profile-and-summary-reports/ quickstats-culture-identity/religion.aspx.

Stout, Y. M., Jacquin, L. A., \& Atwater, P. M. H. (2006). Six major challenges faced by near-death experiencers. Journal of Near-Death Studies, 25, 49-62. doi:10.17514/JNDS-2006-25-1-p49-62.

Sutherland, C. (1990). Changes in religious beliefs, attitudes, and practices following near-death experiences: An Australian study. Journal of Near-Death Studies, 9, 21-31. doi:10.17514/JNDS-1990-9-1-p21-31.

Tassell-Matamua, N. A. (2013). Phenomenology of near-death experiences: An analysis of a Māori case study. Journal of Near-Death Studies, 32, 107117. doi:10.17514/JNDS-1988-6-3-p175-184. doi:10.17514/JNDS-2013-32-2p107-117.

Tassell-Matamua, N. A., \& Murray, M. (2014). Near-death experiences: Quantitative findings from an Aotearoa New Zealand sample. Journal of Near-Death Studies, 33, 2-29. doi:10.17514/JNDS-2014-33-1-p2-29.

Tassell-Matamua, N. A., \& Steadman, K. (2017). "I feel more spiritual". Increased spirituality after a near-death experience. Journal for Studies in Spirituality, 7(1), 35-49.

van Lommel, P. (2010). Consciousness beyond life: The science of the near-death experience. London, England, UK: HarperOne.

van Lommel, P. (2011). Near-death experiences. The experience of the self as 
real and not as an illusion. Annals of the New York Academy of Sciences, 1234, 19-28.

van Lommel, P., van Wees, R., Meyers, V., \& Elferrich, I. (2001). Near death experience in survivors of cardiac arrest: A prospective study in the Netherlands. Lancet, 358, 2039-2045.

Walker, S., Eketone, A., \& Gibbs, A. (2006). An exploration of Kaupapa Māori research, its principles, processes and applications. International Journal of Social Research Methodology, 9, 331-344.

Wilde, D. J., \& Murray, C. D. (2009). The evolving self: Finding meaning in near-death experiences using interpretative phenomenological analysis. Mental Health, Religion and Culture, 12(3), 223-239. 


\section{Appendix A \\ Interview Schedule}

\section{Background}

How old were you when you had your NDE? Do you remember it well?

\section{About your NDE}

Can you tell me about your experience?

- Tell me about the content of your experience in detail.

- Was it positive or negative? How so?

Do you feel like you have experienced significant changes since your NDE?

- Are these due to the fact that you almost died or is it more than that?

\section{Aftereffects}

How has your experience affected you since?

- Has your experience had a significant impact on your life?

- How has your experience changed you?

- How has your experience changed your attitudes and values?

- Has your experience impacted those around you? How?

\section{Relationship between content and life changes}

Do you feel as though any particular part of your NDE contributed to your changes?

- What aspects?

- How did they contribute?

- How do you feel about death and dying now? What made your fear of death decrease? 